\title{
COMPAss: A Space Cognitive Behavior Modeling and Performance Assessment Platform*
}

\author{
Yanfei Liu ${ }^{1}$, Zhiqiang Tian ${ }^{2, * *}$, Yu Zhang ${ }^{1}$, Qi Sun ${ }^{1}$, Junsong Li $^{1}$, \\ Jing $\mathrm{Sun}^{1}$, and Feng $\mathrm{Fu}^{1}$ \\ ${ }^{1}$ Zhejiang Sci-Tech University, Hangzhou, China \\ ${ }^{2}$ China Astronaut Research and Training Center, Beijing, China \\ yliu@zju.edu.cn, tianzhiqiang2000@163.com
}

\begin{abstract}
Based on cognitive architecture, COMPAss (Cognitive behaviOr Modeling and Performance Assessment) -- an integrated research \& development platform oriented space manual control task is proposed. MRvD (Manual rendezvous and docking) control task is selected for cognitive modeling and human performance assessment. The MRvD cognitive behavior model is built on the platform by extract model declarative knowledge, procedural knowledge and model parameters on the basis of experimental data, and the model's validation is verified by comparing the process and results between model's run and actual control. The verification result shows that the model is effective and model's specific parameter can map human certain cognitive characteristic. Finally by comparing model performance with adjusting model's parameter the human performance is evaluated. As an example how skillful degree influence on human performance for MRvD task is evaluated and a report for skillful degree vs. MRvD performance is produced on COMPAss platform.
\end{abstract}

Keywords: Cognitive architecture, Manual rendezvous and docking, Cognitive behavior modeling, Performance assessment.

\section{Introduction}

The rapid and continuous advancement of technology makes the human more likely a limiting factor in system design and performance, makes it increasingly important to consider human factors to optimize usability and safety of systems. As it comes up just after World War II, human factors engineering, along with the closely related disciplines of human-systems integration, human computer interaction, and user-interface design etc., addresses issues of how humans interact with technology and develops rapidly. Over the past decades, the field has grown and diversified into areas such as consumer products, business, highway safety, telecommunications, and, most recently,

\footnotetext{
This work is supported by Zhejiang Provincial Natural Science Foundation under Grant No. LY12C09005, Y1110477, National Natural Science Foundation of China under Grant No.61100183, 6110503 and 973 Program of China under Grant No. 2011CB711000.

** Corresponding author.
} 
health care etc. Especially nowadays, modeling human cognition, and understanding the manner that humans use information, is becoming increasingly important as system designers develop automation to support human operators [1].

Cognitive models are appearing in all fields of cognition at a rapidly increasing rate, and applications of cognitive modeling are beginning to spill over into other fields including human factors, clinical psychology, cognitive neuroscience, agent based modeling in economics, and many more [2]. Cognitive architectures are theories of cognition that try to capture the essential representations and mechanisms that underlie cognition [3]. Research in cognitive architectures has gradually moved from a focus on the functional capabilities of architectures to the ability to model the details of human behavior, and, more recently, brain activity [4]. Some of the most popular architectures for cognitive modeling include ACT-R [5] and Soar etc.

Space exploration began in the second half of the 20th century and rapid developed after latter part of the 70s, its benefits are vital to our rapidly advancing world nowadays, and new space applications are developing more rapidly than ever. However, for astronauts aboard the spaceship/space-station, they are exposed to numerous stressors during spaceflights, such as microgravity, confinement, and radiation, all of which may impair human cognitive capabilities. While some critical operations for spaceflight, such as operating the mechanical arms, extravehicular activities, and driving the spacecraft, etc., fault operation may cause serious disasters. The crewmembers' cognition will affect task's performance, therefor essential spaceflight operation skills must be developed as a team member in an environment of highly dynamic, fast-changing, and even sometimes unpredictable. To improve crewmembers' performance, a lot of research has been conducted [6]. However, due to limitations for experimental conditions, uncertainty and poor features of experimental results in the study of human cognitive behavior for spaceflight, experimental researches are difficult to implement in reality. In the meantime, for the restriction and deficiency of the studies on human mind, using a computer modeling and simulation method to investigate human cognition to improve performance becomes a new method of study on human factors [7].

To explore sophisticated studies on cognitive behavior in complex spaceflight tasks, a practical tool is essential for simulating and analyzing the details of the spaceflight manipulation task. Based on of cognitive architecture, this paper proposes an integrated research \& development platform COMPAss to investigate astronaut's cognitive behavior and improve performance for special spaceflight task.

\section{General Framework of COMPAss}

The platform is designed as three-tier hierarchy to its role in system, it is base layer, function layer and user-interact layer. Figure 1 shows the framework of COMPAss. 

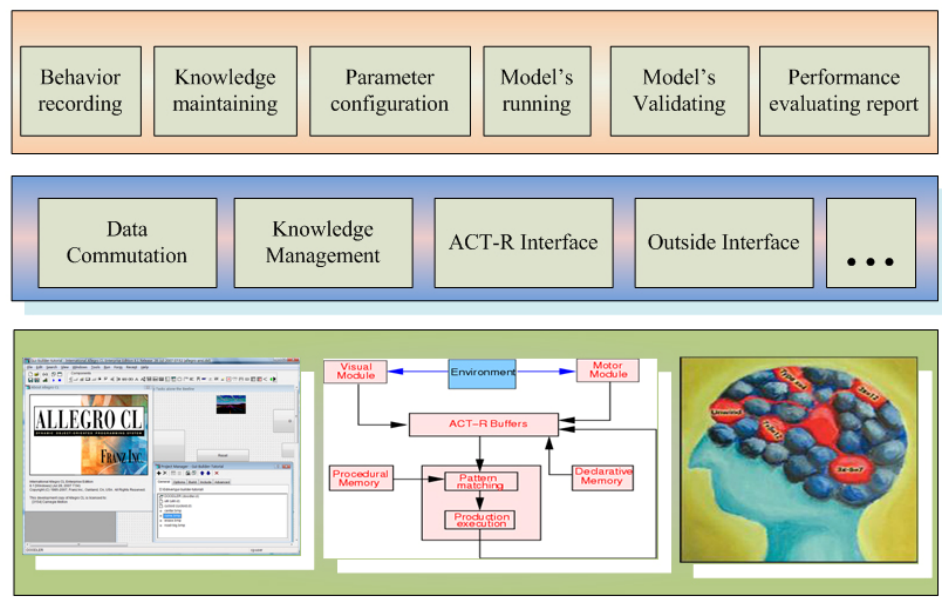

Fig. 1. The hierarchy of the COMPAss platform

The base layer is the cognitive architecture/theory supporting foundation, it includes cognitive architecture running platform, typical cognitive architecture, and facility driver etc. Above the base layer is the functional modules, and it consists of data communication module, knowledge management module, device input/output module and cognitive architecture interface etc. On the top of the functional layer it is application layer. It includes behavior recording, knowledge maintaining, parameter configuration, running \& simulation, model's verification and performance evaluating report generating modules etc.

\section{Modeling and Verification}

\subsection{MRvD Control Task and Behavior Recording}

As a study case spacecraft manual rendezvous and docking (MRvD) control task is selected for cognitive modeling and human performance assessment. The manual control human-machine interface for MRvD is shown in Figure 2, and spacecraft status's information is displayed on the monitor.

During MRvD task, by observing the crosshair-cursor's relative position, the crosshair-cursor's size change, and the crosshair-cursor's motion trend in screen, the operator perceive crosshair-cursor's position, posture and velocity. According to priori knowledge and the information conveyed from perception the operator make response and make decision what measurement it will be taken. If the operator deems it necessary to change the current moving status, manual control operation should to taken and the operator conduct control behavior. After that it will come out a new status, and then 


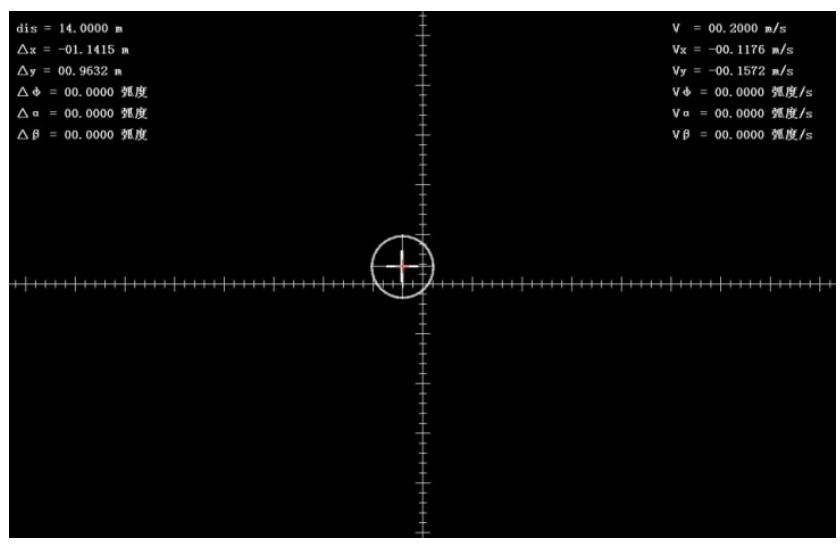

Fig. 2. The manual control human-machine interface for MRvD

a new perception, new decision and control behavior. One after one connective cognitive behavior like these, the operator fulfills the whole MRvD control task. In the course of MRvD mission COMPAss platform record all manual control processes which includes crosshair-cursor position, posture, velocity and operator's manual control behavior according to timeline.

\subsection{MRvD Task Cognitive Modeling}

The most important work of cognitive modeling so far is extraction for declarative knowledge, procedural knowledge and model's parameter. The declarative knowledge is some conceptions definition for the task or some facts, such as the operations, the vehicle's status and the relationship between crosshair-cursor's size and vehicle's distance etc. The procedural knowledge is lots of rules for decision-making of the model. It is acquired by computer aided mining the relationship between cognitive processes and behaviors on the COMPAss platform. Typical procedural knowledge, such as determining operation behavior according the vehicle's location or determining vehicle's distance according to crosshair-cursor's size, are extracted from experimental data. The model parameters are built up according to boundary conditions, some custom constants predefinition and task's characteristics, such as limitation on vehicle's speed and contact speed and maximum allowance of misalignment etc.

With the aid of COMPAss model's declarative knowledge is defined by analyzing experimental record and empirical knowledge; model's procedural knowledge are constructed through extracting relationship between cognitive process and manual control behavior, and model's parameters are refined according to boundary conditions and task's specific characteristics. Based on declarative knowledge, procedural knowledge and model's parameters the COMPAss automatically builds MRvD cognitive behavior model. 


\subsection{Model Verification}

The model's validation is verified from two different stages. The first stage is the model will successfully complete the model's task which model is built based on. The second stage is the model's every single operation while it running coincides with actual cognitive behavior which it appears in experiment in details.

By running different skillful degree operator's MRvD cognitive behavior model on the COMPAss platform, the cognitive process and control behavior will be redisplayed accordingly. Whichever type of the four skillful grade (skilled, less skilled, unskilled and novices), the model can complete the rendezvous and docking task, and the control behavior agree with the actual operation which the model built based on. Figure 3 show the comparison of model's running and human's operation result in spacecraft's longitudinal displacement vs. time. The blue line in figure 3 is the longitudinal distance change with time for model's running, and the black line is from human controlling operations.

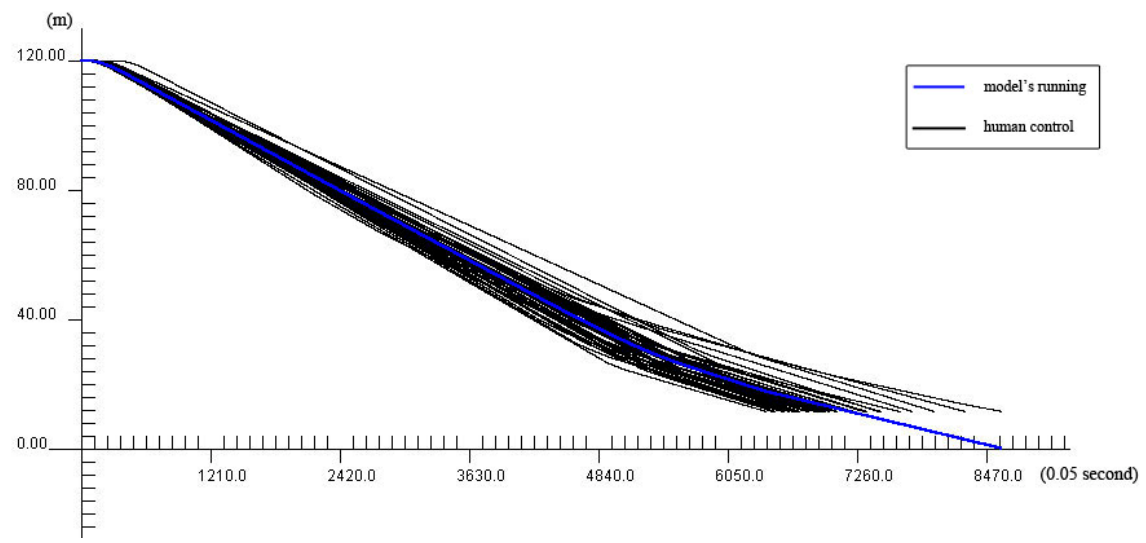

Fig. 3. The comparison of model's running and human's operation

Due to the cognitive architecture vividly portrays human's cognitive process which includes vision perception, information processing, knowledge retrieve, conflict resolution, rules trigger and motor generation, by inspecting the model's cognitive behavior and comparing model's cognition process with operator's cognitive behavior the model based on at a more small time slice (millisecond), the outcome is encouraging.

By comparing the process and results between model running and actual control process the model's validation is verified in two aspects of model accomplishing MRvD task and cognitive behavior details. The result shows that not only the model can fulfill the model's task successfully but also the cognition process and behavior are cohere with real control process. 


\section{$4 \quad$ Human Performance Assessment}

Humans work is within their capabilities, and has reference to physical, cognitive, and perceptual capabilities. Human perceptual capabilities include the abilities to see and to hear. Cognitive capabilities include abilities to reason, remember, communicate, and understand etc.[8] One of the important thing in cognitive behavior modeling is to abstract model parameter to map human's cognitive characteristic. By changing the value of certain cognitive parameter the model will achieve different output. With the impact of specific model's parameter on model's running the influences for cognitive characteristic on behavior can be investigated.

As an example, MRvD task for different skillful operators is selected to study operation performances. By investing the influence of model's parameter's change on model's running result examine how skillful degree influence on human performance for MRvD task and produce the skillful degree vs. MRvD performance report by applying COMPAss platform. Different data set such as the crosshair-cursor's three dimension displacement, velocity and time are collected for different skillful operators. Figure 4 show the performance comparison for skillful and novice operator.

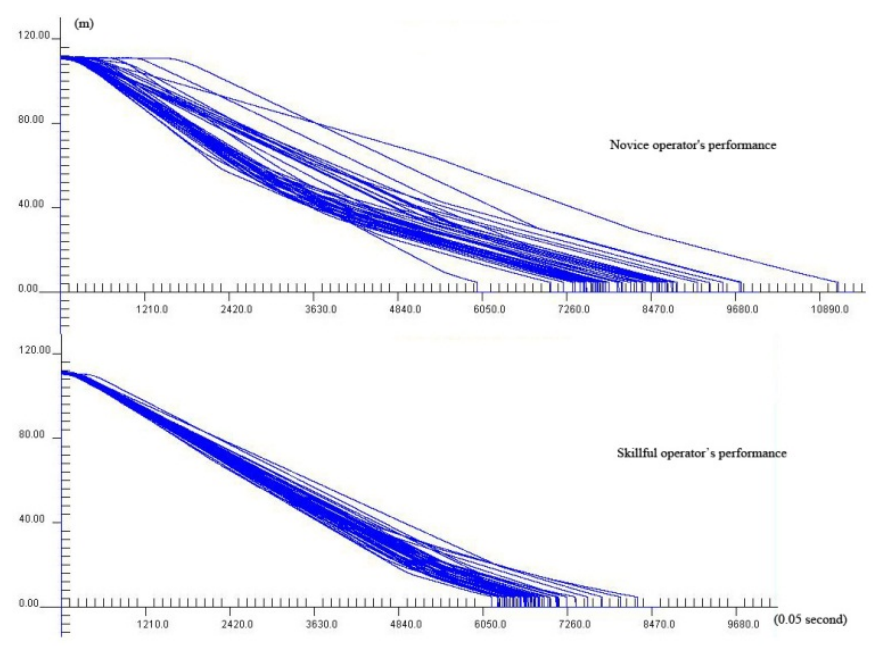

Fig. 1. Performance comparison of skillful and novice operator for MRvD task

By extracting the characteristic of different data set which presented different skillful operators, try to find out what the relationship between characteristic value and skillful degree. Studies show that the procedural knowledge activation base-level constant (:blc) is response to operators' skillful degree. The value of model's parameter :blc for a skilled operator is lower than a novice operator. The further observing find that as value of parameter :blc increase some procedural knowledge are no longer to be retrieved while the model running. 
By adjusting model's parameter and comparing model running consequence under different adjusted parameter, the human performance is evaluated according to certain judging criterion. By combination of related influence of influencing factors and according to the factor's weight in human performance, the human performance assessment method and standard are constructed, and human performance assessment report is generated according to design's purpose.

\section{Conclusion}

This paper's major job is two-folded. Firstly, it proposes and implements a space manipulation task oriented platform for studying human cognitive behavior and human performance assessment. Secondly, a sample for MRvD task is given to detail the whole process of cognitive behavior modeling and manipulation performance's assessment.

\section{References}

1. Borst, J.P., Anderson, J.R.: Using Model-Based functional MRI to locate Working Memory Updates and Declarative Memory Retrievals in the Fronto-Parietal Network. Proceedings of the National Academy of Sciences USA 110(5), 1628-1633 (2013)

2. Busemeyer, J.R., Diederich, A.: Cognitive modeling. Sage (2010)

3. Taatgen, N., Anderson, J.R.: The past, present, and future of cognitive architectures. Topics in Cognitive Science 2(4), 693-704 (2010)

4. Borst, J.P., Anderson, J.R.: Using the ACT-R Cognitive Architecture in combination with fMRI data. In: Forstmann, B.U., Wagenmakers, E.-J. (eds.) An Introduction to Model-Based Cognitive Neuroscience. Springer, New York (2014)

5. Anderson, J.R., Bothell, D., Byrne, M.D., Douglass, S., Lebiere, C., Qin, Y.: An integrated theory of the mind. Psychological Review 111(4), 1036 (2004)

6. Wang, C., Tian, Y., Liu, Y., Chen, S., Tian, Z., Li, J.: Cognitive behavior modeling of manual rendezvous and docking based on the ACT-R cognitive architecture. In: Duffy, V.G. (ed.) HCII 2013 and DHM 2013, Part II. LNCS, vol. 8026, pp. 143-148. Springer, Heidelberg (2013)

7. Lebiere, C., Jentsch, F., Ososky, S.: Cognitive Models of Decision Making Processes for Human-Robot Interaction. In: Shumaker, R. (ed.) VAMR 2013, Part I. LNCS, vol. 8021, pp. 285-294. Springer, Heidelberg (2013)

8. Salvendy, G.: Handbook of human factors and ergonomics (2012), http : / Wiley . com 